\title{
Estudio de la tecnología de concretos de contracción compensada aplicada a la construcción de pisos industriales
}

Study of shrinkage-compensating concrete technology applied to the construction of industrial floors

Fecha de entrega: 5 de diciembre 2012 Fecha de aceptación: 5 de abril 2013

\section{Óscar Gracia ${ }^{1}$, Gonzalo Quesada ${ }^{1}$, Adriana Gómez ${ }^{1}$, Andrés Santacruz ${ }^{2}$ y Liliana Arias $^{3}$}

${ }^{1}$ Grupo de investigación de Estructuras y Construcción, Departamento de Ingeniería Civil, Pontificia Universidad Javeriana, Calle 40 \# 5-50, piso 1, Edificio José Gabriel Maldonado, Bogotá, Colombia, oscargracia@outlook.com, gonzaloquesadab@hotmail.com, adrianagomez@javeriana.edu.co

${ }^{2}$ Cemex Colombia S.A., Calle 99 \# 9A - 54, Edificio 100 Street, torre 3, Piso 7 y 8, Bogotá, Colombia, andresfernando.santacruz@cemex.com

3 Toxement S.A., Calle 20 C No. 43 A 52 - Interior 4, Bogotá, Colombia, lilianaarias@toxement.com.co

Esta investigación consistió en aplicar un Aditivo Compensador de Contracción ACC a una mezcla de concreto para disminuir la fisuración debida a la contracción por secado en la construcción de pisos industriales. Esto con el objeto de construir paños hasta diez veces más grandes del tamaño recomendado $(3 \times 3 \mathrm{~m})$ para un espesor de $15 \mathrm{~cm}$, según las normas ACI 360 y ACI 302. Así se minimiza el número de juntas y los problemas de alabeo, filtraciones de líquidos, fracturas en los bordes e inadecuada transferencia de cargas. La metodología consiste en buscar la dosis óptima de ACC, teniendo como base un diseño de mezcla para un módulo de rotura de $4.2 \mathrm{MPa}$, realizando ensayos de contracción y de expansión restringida con 3 tipos de cemento comerciales colombianos y diferentes porcentajes de dosificación del aditivo. Una vez realizados los ensayos de laboratorio bajo la norma ASTM C 878, se encontró que la dosificación del 9\% de aditivo era la adecuada. Dosificaciones superiores al $11 \%$ generan fisuras internas en el concreto y dosis menores al $9 \%$ no generan la expansión requerida en el concreto para compensar la contracción del mismo. Posterior a ello, se construyó una losa a escala industrial cuyas dimensiones fueron de $9 \times 12 x$ 0.15 m sin juntas, para evaluar el comportamiento del ACC en condiciones de obra. Puesta al servicio la losa siguiendo los procedimientos de curado no se generaron fisuras debidas a contracción por secado, corroborando asi los datos obtenidos en el laboratorio y dejando abierta la posibilidad de hacer paños en losas industriales de mayor dimensionamiento.

Palabras clave: concreto de contracción compensada CCC, pisos industriales, contracción por secado, fisuración, aditivo compensador de contracción ACC
This research involved the application of a shrinkagecompensating additive to a concrete mix, to reduce cracking due to shrinkage by drying in the construction of industrial floors. Thus, panels can be built up to ten times larger than the recommended size $(3 \times 3 \mathrm{~m})$ for a thickness of $15 \mathrm{~cm}$, based on the recommendations of ACI 360 and ACI 302. This can minimize the number of joints and also the problems of warping, leaking water and other liquids, chipping, and inadequate load transfer. The methodology consists in finding the optimum dose of additive, based on a mix design for a rupture modulus of $4.2 \mathrm{MPa}$, subjected to restricted expansion and contraction tests with various types of domestic cements and different percentages of additive dosing. Once all laboratory tests were carried out under the norm ASTM C878, it was found that the dosage of $9 \%$ of additive was adequate. Higher doses produce internal cracks in the concrete, and lower doses did not expand the concrete enough to offset the contraction of it. Following this, a $9 \times 12 \times 0.15 \mathrm{~m}$ slab without joints was built using the optimum shrinkagecompensating additive found in the series of laboratory experiments. After implementing a curing process no cracks were generated during drying, verifying the laboratory results and leaving open the possibility to produce larger panels for industrial floors.

Keywords: shrinkage-compensating concrete, industrial floors, drying shrinkage, cracking, shrinkage- compensating additive 


\section{Introducción}

Los pisos industriales son por lo general estructuras de concreto que tienen características especiales, como su alta planicidad, resistencia mecánica, resistencia química y resistencia al impacto. Estos elementos estructurales deben ser durables y tener un equilibrio entre costo y funcionalidad, dado que son diseñados para industrias que no se pueden detener en la realización de mantenimientos periódicos. Actualmente estos pisos industriales se construyen usando fibras sintéticas o metálicas, acero de refuerzo, aditivos reductores de contracción e incluso se utilizan concretos postensados, para disminuir los problemas causados por los diferentes tipos de contracción. Este artículo trata el tema de la contracción por secado del concreto y se muestran los resultados de una investigación sobre el uso de un Aditivo Compensador de Contracción ACC, el cual expande el concreto disminuyendo la posibilidad de aparición de fisuras ocasionadas por la contracción por secado, permitiendo aumentar el distanciamiento entre juntas.

Sin embargo, los pisos industriales postensados generan altos costos de construcción (torones, gatos hidráulicos, armado de ductos) y exigen la contratación de mano de obra calificada. También se ha probado el uso de macrorefuerzo metálico y/o sintético, lo cual ayuda a disminuir los esfuerzos de contracción en el concreto (Mendoza et al., 2011). Aunque su mayor aporte es el soporte de los esfuerzos por fatiga y postfisuración, en algunos casos con dosificaciones elevadas generando mayores costos y con limitantes como el bombeo, que en la mayoría de los casos no es permitido para concreto con fibras metálicas, por los daños que éstas pueden ocasionar al equipo. Adicionalmente los paños, losas o placas de concreto (estructura horizontal de concreto reforzado utilizada en la construcción de pisos industriales o pavimentos rígidos) logrados sin presencia de fisuras, no son tan extensos como los paños ejecutados con concretos postensados.

En tecnología de concreto se habla de Concreto de Contracción Compensada CCC, que puede ser fabricado con un aditivo tipo $\mathrm{G}$, el cual en combinación con el cemento genera un efecto expansivo en estado fresco y endurecido. Esta expansión minimiza el agrietamiento presentado en la contracción por secado de las losas. En la parte inferior de la Figura 1 se observa el comportamiento volumétrico de un concreto de referencia elaborado con Cemento Portland Ordinario CPO. Igualmente se puede apreciar el comportamiento de un concreto de baja contracción adicionado generalmente con aditivos reductores de contracción y de un CCC adicionado con compensadores de contracción, en donde se puede ver claramente que experimentan una expansión en el volumen de su masa, que es compensada por la contracción del concreto para luego presentar resultados de contracción mínima cercana a cero. La Figura 1 es de tipo esquemática ya que compara los 3 concretos anteriormente descritos de forma proporcional sin el uso de unidades de medida.

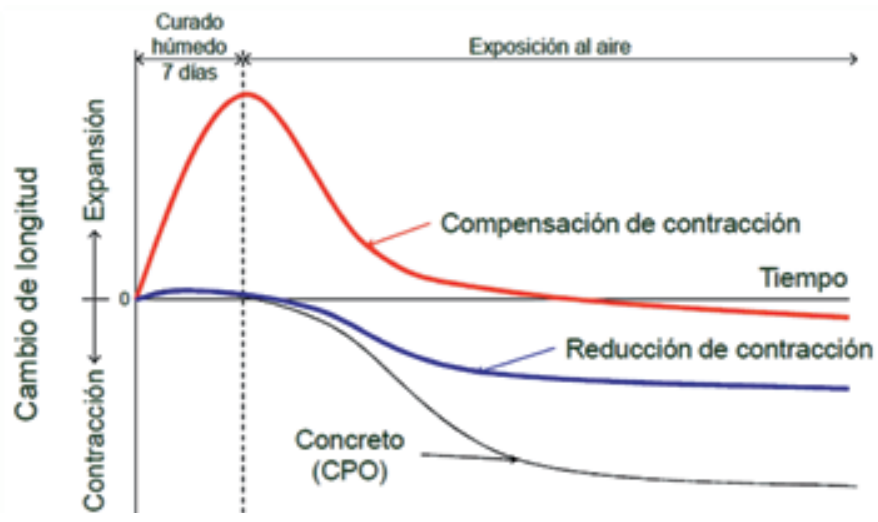

Figura 1: Reducción versus compensación de contracción (ACI 223, 2010).

El efecto expansivo también puede ser logrado con otros tipos de componentes, como los cementos expansivos tipo $\mathrm{K}$, tipo $\mathrm{M}$, tipo $\mathrm{S}$, que básicamente buscan producir etringita con diferentes tipos de mezclas que junto con el cemento y agua reaccionan expansivamente (Monteiro y Mehta, 1986). Estos efectos también se logran con componentes expansivos que igualmente buscan producir etringita o cristales de hidróxido de calcio que incrementan el volumen del concreto significativamente, estos componentes son catalogados según sus componentes y nombrados como tipo $\mathrm{K}, \mathrm{M}, \mathrm{S}$ y $\mathrm{G}$.

Todas las variantes de los aditivos expansores (tipo K, M, S) están basados en la formación de etringita (sulfoaluminato de calcio hidratado) en cantidades considerables durante los primeros días de curado (Nagataki y Gomi, 1998). Esta etringita lo que logra es atraer una gran cantidad de moléculas de agua, que causan una repulsión entre partículas, la cual a su vez produce una sobre expansión de la matriz que le confieren las características de expansión al concreto. 
La expansión potencial producida por la formación de etringita es controlada usando acero de refuerzo común, el acero restringe la expansión global, de tal forma que el acero resiste los esfuerzos a tensión en el concreto y el concreto mismo resiste los esfuerzos a compresión.

En Argentina, se desarrolló un proyecto que consistió en realizar los pisos industriales de un centro de distribución de supermercados. Los parámetros de diseño tenían en cuenta el uso de estanterías de mucha altura, auto elevadores computarizados, y otros aspectos técnicos de alto nivel, que requieren minimizar las juntas y lograr alta planicidad, evitando la aparición de fisuras y el alabeo de las losas. En este proyecto se utilizó un aditivo de retracción compensada, a base de Sulfoaluminato de Calcio y Óxido de Calcio (Luco et al., 2003).

En Colombia se busca implementar esta tecnología evaluando sus características y comportamiento con los cementos locales. El presente trabajo documenta la investigación realizada con el fin de comprobar la compatibilidad y efectividad del ACC con tres cementos evaluados cuyas características se describen más adelante, buscando implementar dicha tecnología. Se realizaron una serie de ensayos de laboratorio enfocados a demostrar si las características planteadas por el fabricante eran válidas para su implementación en la construcción de pisos industriales. Finalmente, se presentan los resultados obtenidos, recomendaciones y conclusiones.

\section{Objetivos de la investigación}

Evaluar la aplicabilidad de un ACC en los concretos diseñados para un Módulo de Rotura MR de 4.2 MPa, utilizados en la construcción de pisos industriales de gran formato. Comprobar la expansión del concreto al aplicarle un ACC en una dosis adecuada, para así lograr controlar la fisuración generada en la etapa de contracción por secado y de esta forma poder realizar losas de dimensiones hasta 10 veces mayores a las utilizadas normalmente $(3 \times 3 \mathrm{~m})$ para la construcción de pisos industriales. Diseñar una mezcla de concreto con MR 4.2 MPa, para la optimización de un piso industrial, utilizando un ACC y validar los resultados obtenidos en el laboratorio con una prueba a nivel industrial, usando el diseño de mezcla óptimo.

\section{Metodología}

\section{Diseño de mezcla tipo}

Se diseñó una mezcla de concreto para piso industrial, teniendo como base un MR de 4.2 MPa, que se usó como diseño tipo durante la investigación, bajo los estándares de la norma ACI 302 (2004). Para controlar las variables solo se modificó la cantidad de ACC en la mezcla de concreto. Se incluyeron ensayos adicionales para ajustar el diseño, y así verificar la eficiencia de la mezcla de concreto. Se realizaron 6 probetas de $150 \times 150$ × $530 \mathrm{~mm}$ (vigas para MR), a las cuales se les realizó ensayo de flexión a 3, 7 y 28 días. La granulometría de los agregados pétreos usados se muestra en la Tabla 1. Cabe anotar que el material fue obtenido directamente del almacenamiento en cantera. Para controlar la humedad de los agregados se realizaron pruebas de absorción y posterior corrección de humedad para no alterar las características de las mezclas.

Tabla 1: Granulometría de los agregados usados en la investigación

\begin{tabular}{ccccc}
\hline \multicolumn{2}{c}{ Tamiz } & \multicolumn{3}{c}{ Material } \\
\hline Malla & abertura, & grava & arena \\
& & & grava 12,5 & \\
ASTM & mm & $25 \mathrm{~mm}$ & & triturada \\
\hline 1 & 25 & 86,1 & 100,0 & 100,0 \\
$3 / 4$ & 19 & 26,4 & 100,0 & 100,0 \\
$1 / 2$ & 12,5 & 2,7 & 89,6 & 100,0 \\
$3 / 8$ & 9,5 & 1,5 & 63,4 & 99,5 \\
$\mathrm{~N}^{\circ} 4$ & 4,75 & 1,4 & 10,6 & 91,4 \\
$\mathrm{~N}^{\circ} 8$ & 2,36 & 1,4 & 1,8 & 69,2 \\
$\mathrm{~N}^{\circ} 16$ & 1,18 & 1,4 & 1,8 & 56,9 \\
$\mathrm{~N}^{\circ} 30$ & 0,6 & 1,4 & 1,8 & 49,8 \\
$\mathrm{~N}^{0} 50$ & 0,3 & 1,4 & 1,8 & 38,4 \\
$\mathrm{~N}^{\circ} 100$ & 0,15 & 1,4 & 1,8 & 22,5 \\
$\mathrm{~N}^{\circ} 200$ & 0,075 & 1,4 & 0,3 & 15,5 \\
\hline
\end{tabular}

Se propuso un diseño de mezcla el cual se procedió a validar ejecutando ensayos de flexión y contracción del concreto. En la Tabla 2 se muestran las características de los materiales empleados.

El cemento establecido para la investigación es tipo I (NTC $2871,2004)$, dado que es el más usado para la construcción de pisos industriales. Aunque la norma técnica colombiana tiene como antecedente la norma ASTM C150 (2012), los cementos en Colombia son adicionados con cantidades 
superiores al $5 \%$ establecido en la norma original. Las adiciones utilizadas en Colombia son: cenizas, calizas, escoria de alto horno, entre otros.

Tabla 2: Materiales y propiedades

\begin{tabular}{ccccc}
\hline Material & tamaño, mm & origen & $\begin{array}{c}\text { densidad, } \\
\mathrm{kg} / 1\end{array}$ & absorción, \% \\
\hline Grava & 25 & Bogotá & 2,46 & 1,9 \\
Grava & 12.5 & Bogotá & 2,54 & 1,31 \\
Arena & 5 & Bogotá & 2,73 & 1,67 \\
Cemento & N/A & ---- & $3-3,05$ & ---- \\
\hline
\end{tabular}

Se hizo un muestreo de este tipo de cemento, incluyendo los tres principales productores de este material en Colombia. Para los tres casos, se establece que los sistemas cemento y ACC tipo G son compatibles. En la Tabla 3 se aprecia la composición mineralógica de dichos cementos. Se evaluaron 3 cementos de las casas más utilizados en Colombia, los cuales se nombrarán en el documento como $\mathrm{C} 1, \mathrm{C} 2$ y C3.

Tabla 3: Composición mineralógica de los cementos en evaluación

\begin{tabular}{lccc}
\hline \multirow{2}{*}{ Minerales } & Cemento C1 & Cemento C2 & Cemento C3 \\
\cline { 2 - 4 } & $\%$ masa & \% masa & \% masa \\
\hline $\mathrm{CaO}$ & 56,44 & 55,24 & 60,95 \\
$\mathrm{SiO}_{2}$ & 17,16 & 18,34 & 23,04 \\
$\mathrm{Al}_{2} \mathrm{O}_{3}$ & 3,87 & 5,12 & 7,11 \\
$\mathrm{Fe}_{2} \mathrm{O}_{3}$ & 2,58 & 3,91 & 2,61 \\
$\mathrm{MgO}$ & 2,49 & 1,06 & 1,16 \\
$\mathrm{SO}_{3}$ & 3,42 & 2,92 & 2,57 \\
$\mathrm{~K}_{2} \mathrm{O}$ & 0,48 & 0,61 & 0,61 \\
$\mathrm{P}_{2} \mathrm{O}_{5}$ & 0,11 & 0,26 & 0,21 \\
$\mathrm{TiO}_{2}$ & 0,19 & 0,28 & 0,33 \\
$\mathrm{Na}_{2} \mathrm{O}$ & 0,89 & 0,20 & 0,12 \\
$\mathrm{Cr}_{2} \mathrm{O}_{3}$ & 0,00 & 0,00 & 0,00 \\
$\mathrm{SrO}_{\mathrm{ZnO}}$ & 0,32 & 0,07 & 0,09 \\
$\mathrm{Mn}_{2} \mathrm{O}_{3}$ & 0,01 & 0,01 & 0,02 \\
$\mathrm{LOI}$ & 0,05 & 0,12 & 0,16 \\
$\mathrm{EqAlkali}_{3}$ & 2,50 & 2,37 & 5,60 \\
$\mathrm{C}_{3} \mathrm{~S}$ & 1,21 & 0,60 & 0,52 \\
$\mathrm{C}_{2} \mathrm{~S}$ & 59,91 & 37,18 & 14,20 \\
$\mathrm{C}_{3} \mathrm{~A}$ & 4,00 & 24,53 & 55,35 \\
$\mathrm{C}_{4} \mathrm{AF}$ & 5,89 & 6,95 & 14,43 \\
\hline & 8,85 & 11,90 & 7,94 \\
\hline
\end{tabular}

Con estos cementos se realizó la validación de diseño de mezcla para los 3 tipos de cemento (Tabla 4); este diseño fue optimizado bajo parámetros de concretos de baja contracción y siguiendo los lineamientos de diseño de la norma ACI 301 (2010) y ACI 360 (2010). Con este diseño se llegó a un MR de 5,15 MPa a 28 días superando el valor calculado (promedio de las 3 mezclas realizadas). Posterior a ello, se realizó la caracterización mecánica de la mezcla para cada uno de los cementos en estudio.

Tabla 4: Diseño de mezcla tipo propuesto para un $\mathrm{m}^{3}$

\begin{tabular}{lccccccc}
\hline Material & $\begin{array}{c}\text { grava } \\
\mathrm{kg} / \mathrm{m}^{3}\end{array}$ & $\begin{array}{c}\text { grava } \\
25 \mathrm{~mm}, \\
\mathrm{~kg} / \mathrm{m}^{3}\end{array}$ & $\begin{array}{c}12,5 \\
\mathrm{~mm}, \mathrm{~m}^{3}\end{array}$ & $\begin{array}{c}\text { arena, } \\
\mathrm{kg} / \mathrm{m}^{3}\end{array}$ & $\begin{array}{c}\text { agua, } \\
1\end{array}$ & $\begin{array}{c}\text { reductor } \\
\text { de agua, } \\
\mathrm{kg} / \mathrm{m}^{3}\end{array}$ & $\begin{array}{c}\text { rela- } \\
\text { ción } \\
\mathrm{A} / \mathrm{C}\end{array}$ \\
\hline Cantidad & 400 & 720 & 320 & 740 & 176 & $\begin{array}{c}0,76 \\
(0,2 \%)\end{array}$ & 0,44 \\
\hline
\end{tabular}

\section{Caracterización de la mezcla}

Para llevar a cabo la presente etapa se construyeron 6 unidades de vigas para MR y 3 unidades de probetas de 101 x 101 x 254 mm (vigas de contracción), con el diseño tipo, para cada uno de los tres cementos en estudio. Con dichas muestras se determinó MR y el módulo de contracción para cada uno de los cementos evaluados. Las vigas para MR se fallaron a 3, 7 y 28 días, mientras que las vigas de contracción se midieron a lo largo de 56 días, todo bajo los estándares de las normas de contracción del concreto ASTM C157 (2008) y MR en vigas con carga en los tercios NTC 2871 (2004). Se ajusta el contenido de cemento en el diseño (Tabla 5) a $380 \mathrm{~kg} / \mathrm{m}^{3}$, buscando reducir el contenido de pasta y la contracción en el concreto.

Tabla 5: Diseño final de mezcla propuesto para un $\mathrm{m}^{3}$ de concreto

\begin{tabular}{cccccccc}
\hline \multirow{2}{*}{ Material } & $\begin{array}{c}\text { grava } \\
\mathrm{kg} / \mathrm{m}^{3}\end{array}$ & $\begin{array}{c}\text { grava } \\
12,5 \mathrm{~mm}, \\
\mathrm{~kg} / \mathrm{m}^{3}\end{array}$ & $\begin{array}{c}\mathrm{mm}, \\
\mathrm{kg} / \mathrm{m}^{3}\end{array}$ & $\begin{array}{c}\text { arena } \\
\mathrm{kg} / \mathrm{m}^{3}\end{array}$ & $\begin{array}{c}\text { agua, } \\
1\end{array}$ & $\begin{array}{c}\text { reductor } \\
\text { de ce- } \\
\text { mento }\end{array}$ & $\begin{array}{c}\text { rela- } \\
\text { ción } \\
\mathrm{A} / \mathrm{C}\end{array}$ \\
\hline Cantidad & 380 & 720 & 320 & 740 & 167,2 & 0,2 & 0,44 \\
\hline
\end{tabular}

Se procedió a caracterizar cada uno de los cementos en cuanto a su resistencia a flexión (Figura 2) y contracción (Figura 3), ya que éstas son las características principales a evaluar para el diseño de mezcla. 


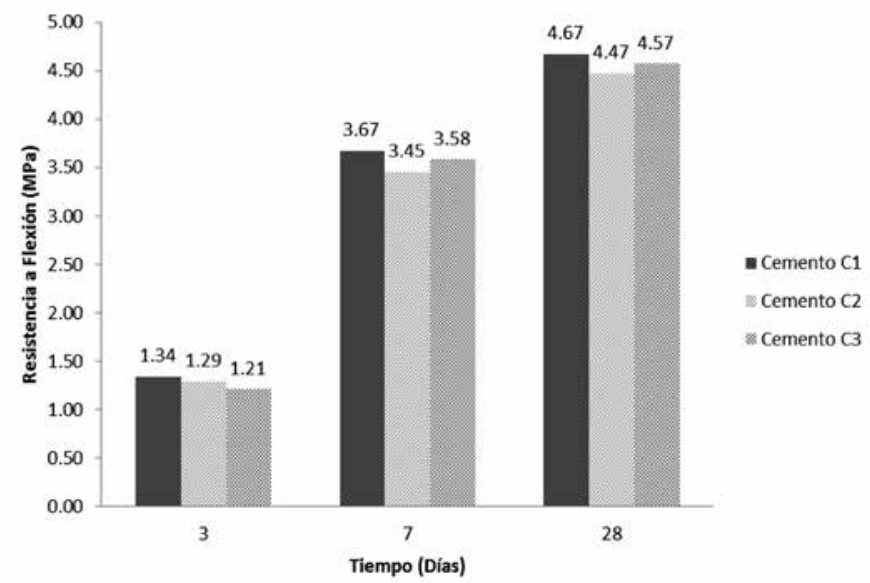

Figura 2: Resistencia a la flexión de cementos de prueba

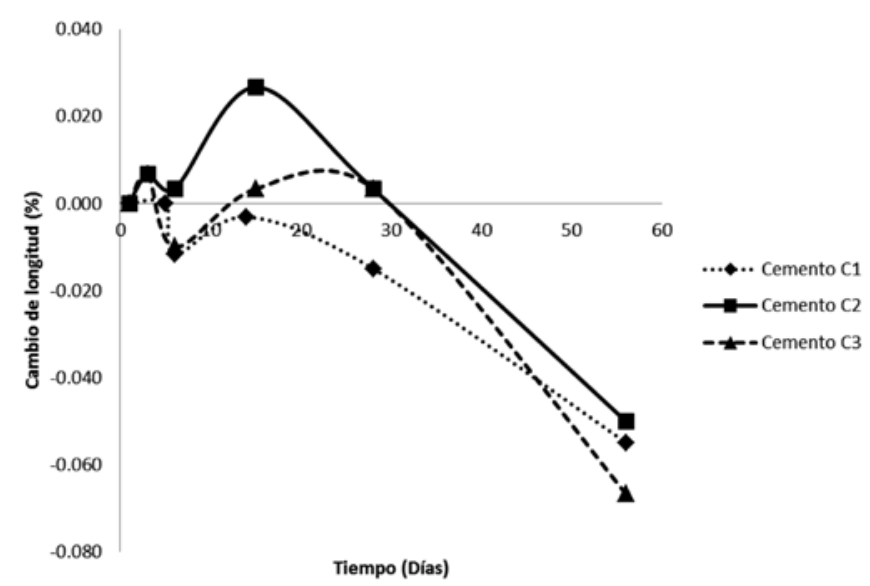

Figura 3: Resultado de contracciones de acuerdo con ASTM C157 (2008)

\section{Ajuste dosificación - Aditivo Compensador de Contracción ACC}

Los componentes que integran el ACC empleado son: óxido de calcio, cemento portland, cenizas volantes, sílice cristalina, arena y lignosulfonato de sodio. Igualmente para el aditivo reductor de agua empleado sus componentes son: agua, sulfonato de naftaleno y sulfonato de sodio. Modificando el porcentaje del aditivo según el peso del cemento por $\mathrm{m}^{3}$ de mezcla, entre los valores recomendados por el fabricante $(9,10,11$ y $12 \%)$, se realizaron ensayos con los tres cementos empleados en la investigación, y basados en los resultados arrojados, se determinó la curva de expansión y contracción de cada uno de los concretos.

Para medir el cambio volumétrico se realizaron ensayos de expansión restringida según la norma ASTM C878 (2009). Se construyeron 3 unidades de vigas de 76 × 76 × $254 \mathrm{~mm}$ (vigas de expansión restringida), usando las restricciones metálicas estipuladas en la norma, por cada porcentaje de dosificación y para cada uno de los 3 cementos evaluados.

Las pruebas correspondientes al ACC, se realizaron variando el porcentaje de éste, para cada uno de los cementos. Para tener un punto de comparación se realiza una mezcla testigo sin ACC; el diseño de mezcla evaluado se muestra en la Tabla 6 . Se aumentó en $0.3 \%$ el aditivo reductor de agua en la mezcla con ACC para lograr el asentamiento requerido, ya que como su presentación es en polvo, éste reduce la manejabilidad del concreto.

Tabla 6: Diseño de mezcla en estado saturado superficialmente seco

\begin{tabular}{lcc}
\hline Material & $\sin$ ACC & con ACC \\
\hline Cemento, $\mathrm{kg} / \mathrm{m}^{3}$ & 380 & 380 \\
Grava $25 \mathrm{~mm}, \mathrm{~kg} / \mathrm{m}^{3}$ & 720 & 720 \\
Grava $12,5 \mathrm{~mm}, \mathrm{~kg} / \mathrm{m}^{3}$ & 320 & 320 \\
Arena, $\mathrm{kg} / \mathrm{m}^{3}$ & 740 & 740 \\
Agua, 1 & 167,2 & 167,2 \\
Reductor de agua, \% cemento & 0,2 & 0,5 \\
Relación A/C & 0,44 & 0,44 \\
\hline
\end{tabular}

Para poder ejecutar los ensayos de expansión restringida, fue necesario conocer de antemano los tiempos de fraguado (Figura 4) con el objeto de establecer el tiempo de retiro de los moldes de las muestras y la lectura inicial de expansión con la cual se va a comparar las medidas siguientes, para así conocer el cambio volumétrico presentado en el tiempo.

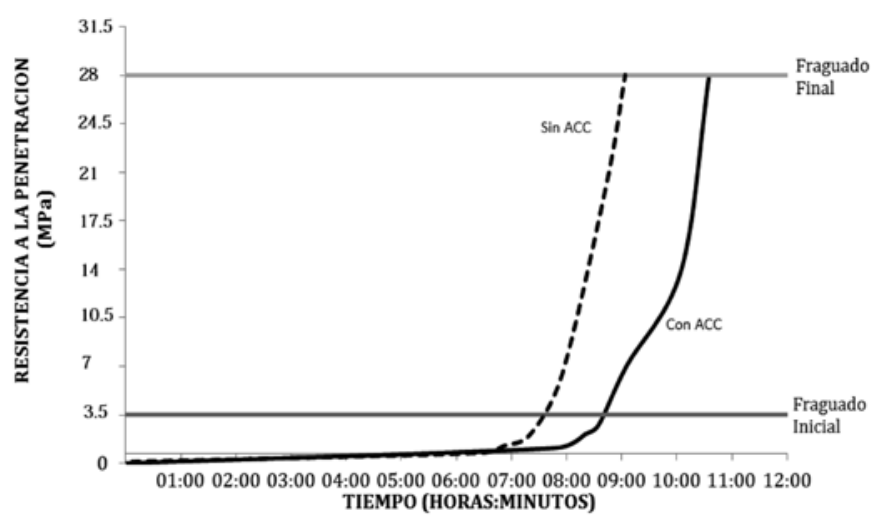

Figura 4: Resultados de los tiempos de fraguado sin ACC y con ACC.

Como resultado de estos ensayos se establece que la mezcla con ACC presenta un tiempo de fraguado de dos horas más que la mezcla de referencia sin aditivo. Una 
vez tomados los tiempos de fraguado, se procede con la toma de muestras de los ensayos de expansión restringida. Los resultados obtenidos en los ensayos mencionados se pueden observar en las Figuras 5, 6 y 7 para C1, C2 y C3 respectivamente. La mezcla con el $12 \%$ de ACC presentó cambios volumétricos considerables lo que provocó que la probeta de ensayo se fracturara y no se pudiera continuar midiendo. Debido a esto se optó por no considerar muestras con sobredosificación de aditivo ACC de $12 \%$ o más.

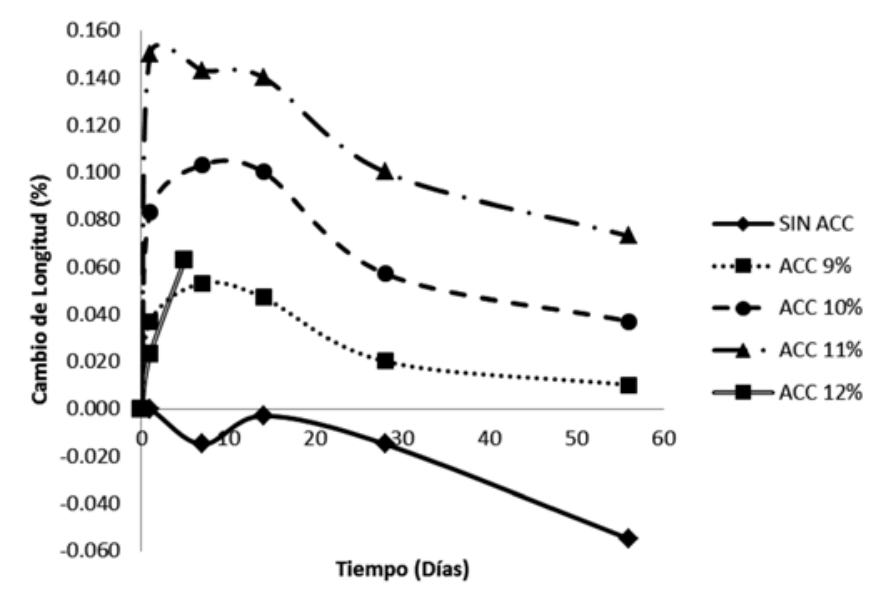

Figura 5: Variación de longitud en el tiempo para cemento C1

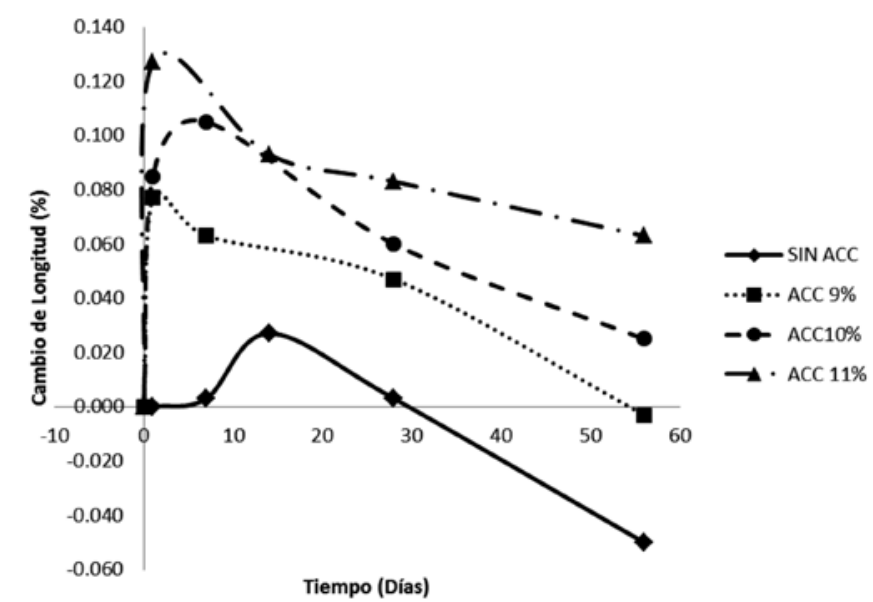

Figura 6: Variación de longitud en el tiempo para cemento C2

\section{Diseño de mezcla - Concreto de Contracción Compen- sada CCC}

Teniendo el porcentaje ideal de ACC por $\mathrm{m}^{3}$ de concreto, según los requerimientos de expansión de la mezcla, se procede a diseñar un concreto de Contracción Compensada CCC bajo la guía ACI 223 (2010), usando el ACC (Figura 8). Se efectuaron pruebas para determinar la eficiencia y comportamiento de dicha mezcla usando 2 unidades de vigas de MR falladas a 28 días y 3 unidades de vigas de expansión restringida tomando medidas a lo largo de 56 días.

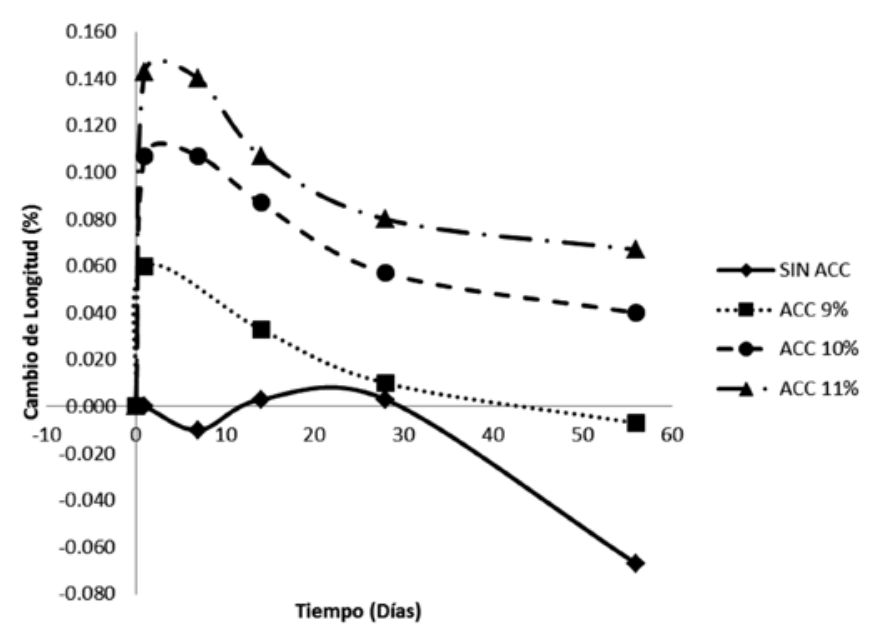

Figura 7: Variación de longitud en el tiempo para cemento C3

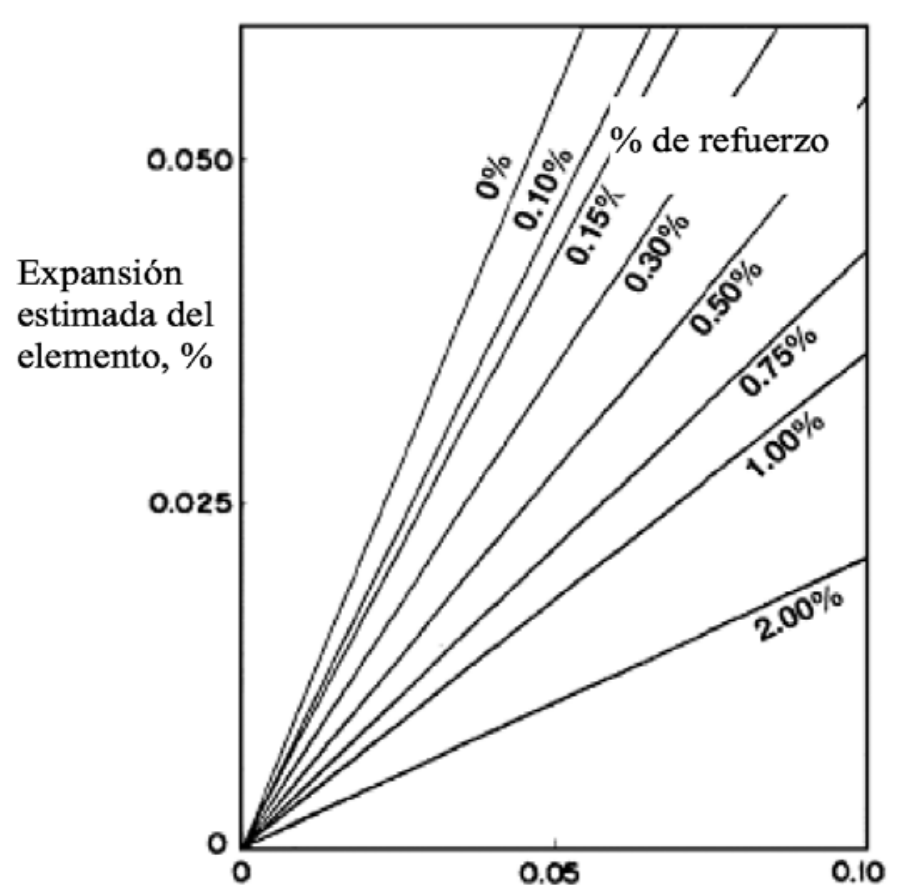

Expansión máxima del prisma de concreto restringido, \% (ASTM C878)

Figura 8: Prisma de expansión, según la contracción esperada y porcentaje de acero de refuerzo para un sistema con componente tipo G (ACI 223, 2010)

La contracción del cemento $\mathrm{C} 1$ se puede compensar con una adición del 9\% de ACC; dado que al entrar los datos de 
expansión estimada en el prisma de expansión requerida (Figura 8), este porcentaje es el que mejor se acopla al valor de expansión requerida. Esta dosificación igualmente se valida con las mediciones tomadas hasta 56 días dado que la contracción es cercana a cero; lo cual significa que el elemento no presentará contracción o su tendencia será cercana a cero cumpliendo con la hipótesis planteada. Las dosificaciones de $10 \%$ y $11 \%$ presentan una expansión superior a la requerida para compensar la contracción en el concreto evaluado, esto genera efectos no controlados en la masa de concreto afectando las consideraciones de diseño y de comportamiento de la losa de concreto.

Aunque los valores de contracción para los tres diseños de mezcla evaluados con tres cementos son diferentes respecto a la compensación, al igual que el cemento $\mathrm{C} 1$, el porcentaje adecuado para dosificar la mezcla de concreto para piso industrial con $\mathrm{C} 2$ y C3 y compensar la contracción de dicha mezcla de concreto es $9 \%$.

Basados en los resultados recogidos en la etapa experimental anterior y la guía ACI 223 (2010), se realiza una repetición de la mezcla que se estima es la más adecuada, evaluando resistencia a flexión, contracción y expansión (Tablas 7, 8 y 9).

Las imágenes de probetas y ensayos realizados en el laboratorio se pueden observar en la Figura 9.

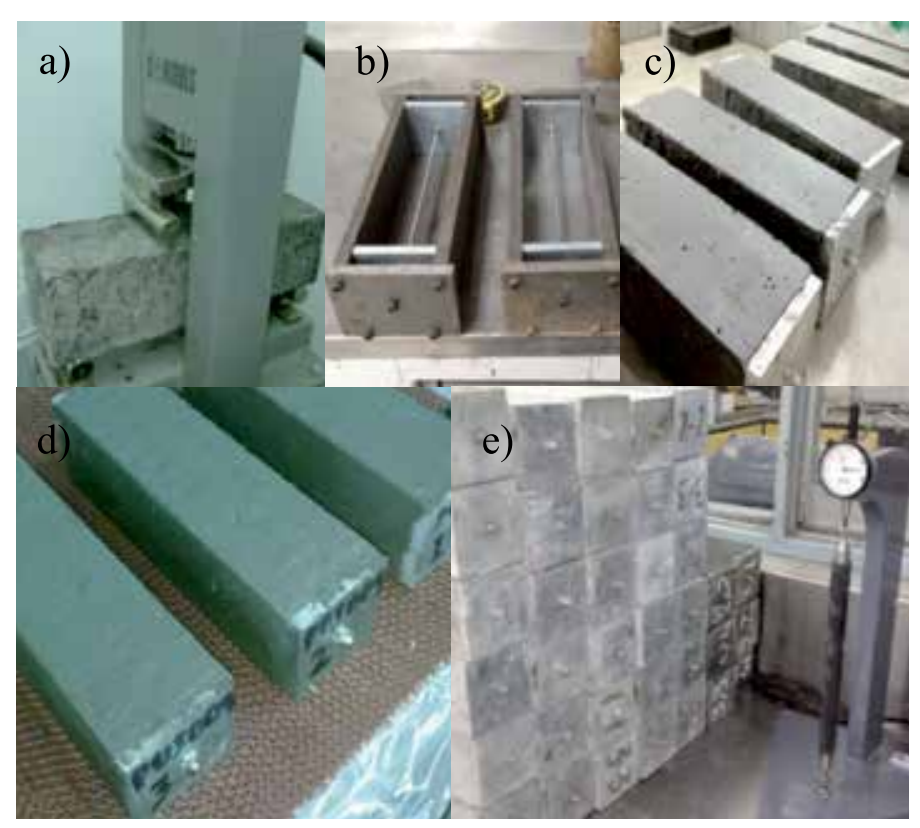

Figura 9. a) Equipo de ensayo de flexotracción, b) moldes de preparación de muestras, c) y d) probetas preparadas y e) equipo para determinación de dilatación restringida del concreto
Tabla 7: Resultados estandarización mezcla C1 y ACC

\begin{tabular}{lccc}
\hline Resultados & Unidades & sin ACC & ACC 9\% \\
\hline $\begin{array}{l}\text { Asentamiento } \\
\text { Resistencia a la flexión a } \\
28 \text { días }\end{array}$ & $\mathrm{cm}$ & 10,32 & 8,918 \\
$\begin{array}{l}\text { Expansión max. ASTM } \\
\text { C878 }\end{array}$ & $\%$ & 4,57 & 5,15 \\
$\begin{array}{l}\text { Expansión 56 días } \\
\text { ASTM C878 }\end{array}$ & $\%$ & $\mathrm{NA}$ & 0,053 \\
$\begin{array}{l}\text { Contracción a 56 días } \\
\text { ASTM C157 }\end{array}$ & $\%$ & $-0,055$ & NA \\
\hline
\end{tabular}

Tabla 8: Resultados estandarización mezcla C2 y ACC

\begin{tabular}{|c|c|c|c|}
\hline Resultados & Unidades & $\sin \mathrm{ACC}$ & ACC $9 \%$ \\
\hline Asentamiento & $\mathrm{cm}$ & 10,95 & 9,362 \\
\hline $\begin{array}{l}\text { Resistencia a la flexión } \\
\text { a } 28 \text { días }\end{array}$ & $\mathrm{MPa}$ & 4,25 & 5,53 \\
\hline $\begin{array}{l}\text { Expansión max. ASTM } \\
\text { C878 }\end{array}$ & $\%$ & NA & 0,077 \\
\hline $\begin{array}{l}\text { Expansión } 56 \text { días } \\
\text { ASTM C } 878\end{array}$ & $\%$ & NA & 0,003 \\
\hline $\begin{array}{l}\text { Contracción a } 56 \text { días } \\
\text { ASTM C } 157\end{array}$ & $\%$ & $-0,050$ & NA \\
\hline
\end{tabular}

Tabla 9. Resultados estandarización mezcla C3 y ACC

\begin{tabular}{lccc}
\hline Resultados & Unidades & $\sin$ ACC & ACC 9\% \\
\hline $\begin{array}{l}\text { Asentamiento } \\
\text { Resistencia a la flexión }\end{array}$ & $\mathrm{cm}$ & 10,78 & 9,316 \\
$\begin{array}{l}\text { a 28 días } \\
\text { Expansión max. ASTM }\end{array}$ & $\%$ & 4,48 & 5,56 \\
$\begin{array}{l}\text { C878 } \\
\begin{array}{l}\text { Expansión 56 días } \\
\text { ASTM C878 }\end{array}\end{array}$ & $\%$ & NA & 0,060 \\
$\begin{array}{l}\text { Contracción a 56 días } \\
\text { ASTM C157 }\end{array}$ & $\%$ & $-0,067$ & NA \\
\hline
\end{tabular}

\section{Prueba piloto}

Con las lecciones aprendidas en el laboratorio se llevó a cabo una prueba a escala industrial. Las dimensiones del piso construido en la prueba piloto fueron de $9 \times 12 \times 0.15$ m, para mayor aplicabilidad de los resultados se ejecutó la prueba utilizando un diseño de mezcla comercial para pisos industriales (Tabla 10). 
Las etapas de construcción de la prueba piloto se aprecian en la secuencia de imágenes mostrada en la Figura 10.

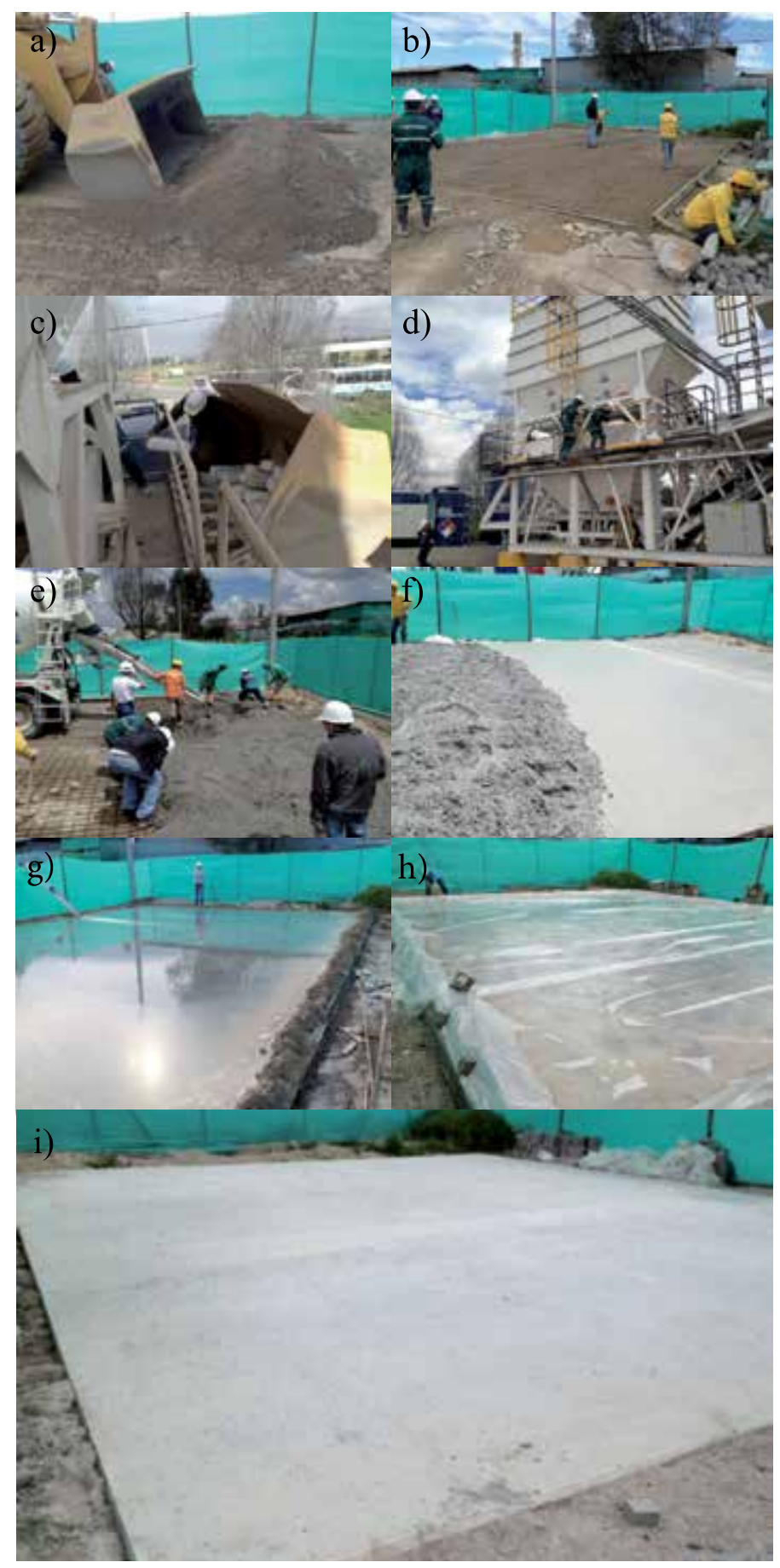

Figura 10: Imágenes del proceso constructivo de la prueba piloto, a) preparación del terreno, b) colocación de moldaje perimetral, c) descarga de cemento, d) planta de concreto, e) vertido de concreto, f) nivelado, g) piscina de saturación, h) retención de la humedad e i) estado final
Tabla 10: Diseño de mezcla prueba piloto

\begin{tabular}{|c|c|c|c|c|c|c|c|}
\hline $\begin{array}{c}\text { cemento } \\
\mathrm{kg} / \mathrm{m}^{3}\end{array}$ & $\begin{array}{c}\text { grava } 25 \\
\mathrm{~mm}, \\
\mathrm{~kg} / \mathrm{m}^{3}\end{array}$ & $\begin{array}{c}\text { grava } \\
12,5 \mathrm{~mm} \\
\mathrm{~kg} / \mathrm{m}^{3}\end{array}$ & $\begin{array}{l}\text { arena, } \\
\mathrm{kg} / \mathrm{m}^{3}\end{array}$ & $\begin{array}{c}\text { agua, } \\
1\end{array}$ & $\begin{array}{l}\text { reductor } \\
\text { de agua, \% } \\
\text { cemento }\end{array}$ & $\begin{array}{l}\text { relación } \\
\mathrm{A} / \mathrm{C}\end{array}$ & $\begin{array}{c}\mathrm{ACC} \\
9 \%, \\
\mathrm{~kg} / \mathrm{m}^{3}\end{array}$ \\
\hline 395 & 805 & 185 & 760 & 170 & 0,86 & 0,44 & 35.55 \\
\hline
\end{tabular}

Se realizaron ensayos de flexión (Figura 11) y compresión para verificar la resistencia de la mezcla de concreto ejecutada. Según los datos obtenidos, a los 15 días de haber realizado el vaciado del concreto se tenía más de un $100 \%$ de la resistencia calculada en el diseño (MR 4.1 $\mathrm{MPa})$. A los 28 días se presentó una resistencia superior al $140 \%$ del valor calculado, lo cual verifica que el concreto con ACC aumenta su resistencia de diseño.

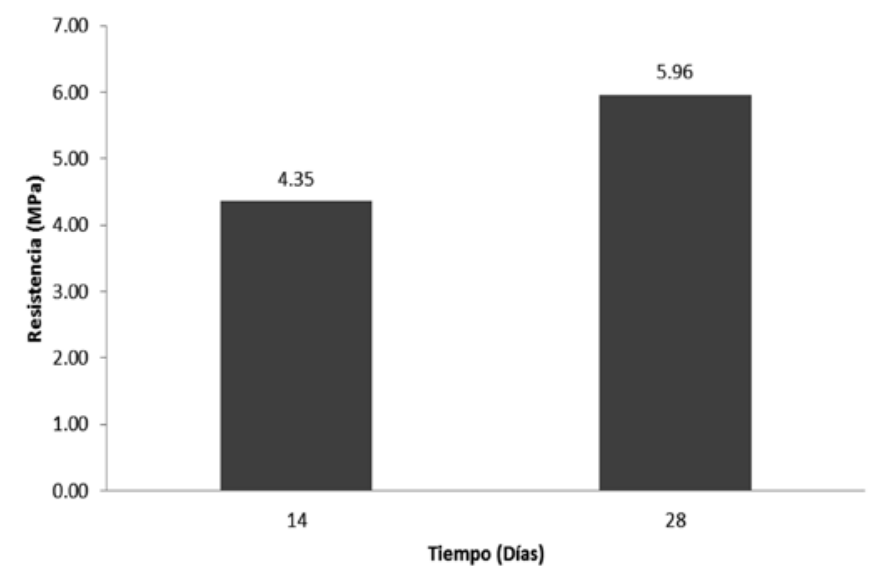

Figura 11: Resultados ensayo de MR (resistencia a la flexión) muestras prueba piloto (MR diseño 4.1 MPa)

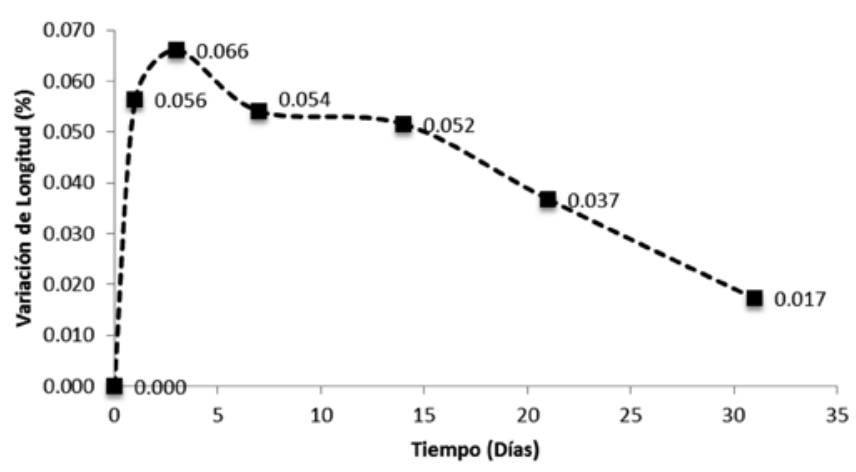

Figura 12: Variación de longitud en el tiempo del concreto de la prueba piloto

En la Figura 12 se aprecian los datos tomados para llevar un control del comportamiento del ACC en la prueba piloto. La expansión que se observó fue la esperada según 
las mediciones obtenidas en las pruebas de laboratorio realizadas anteriormente. Comparada con los resultados de las Figuras 5, 6 y 7, se observa que la conducta es equiparable a lo registrado en el laboratorio en cuanto a las adiciones de ACC al 9\%. Para la contracción evaluada del concreto que se usó en la prueba piloto, los datos de expansión que se debía presentar de acuerdo con la Figura 8 se encuentran dentro del rango aceptable según la guía ACI 223 (2010) en cuanto a expansiones máximas respecto a la contracción registrada.

A lo largo de todas las inspecciones visuales realizadas durante 28 días, luego de ser ejecutado el vaciado del concreto, no se observaron fisuras en la losa de concreto (Figura 13), motivo por el cual se puede afirmar que la prueba realizada arrojó resultados positivos, junto con los resultados obtenidos de los análisis de laboratorio, en cuanto a variación de la longitud de las muestras y la resistencia a la flexión MR, las cuales superaron ampliamente el diseño propuesto.

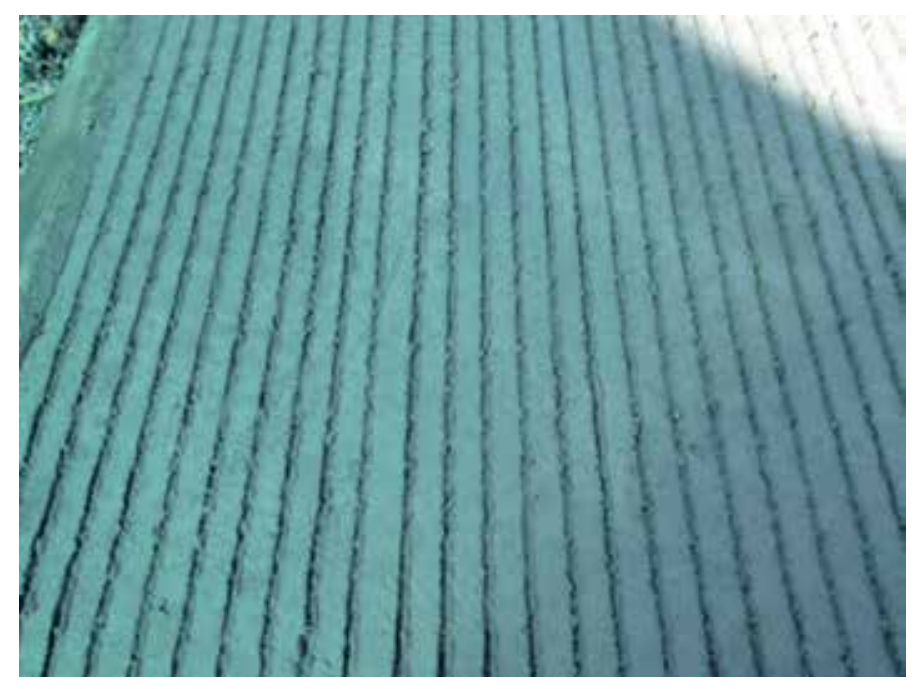

Figura 13: Inspección visual prueba piloto

\section{Discusión}

En Argentina en los pisos del Centro de Distribución de los supermercados COTO ubicado en los alrededores de Buenos Aires, con un aditivo compensador de contracción al 10\% lograron los resultados deseados, pero observaron que por las adiciones de sus cementos, mayores adiciones de ACC no tenían variación, caso contrario a los cementos colombianos que a mayor proporción de aditivo aumentaba considerablemente la expansión. Ello conlleva a que dentro de la masa de concreto se generen esfuerzos y se pueden crear fisuras que afecten la resistencia y propiedades mecánicas de las losas del piso industrial. Igualmente, en Argentina no se observó incremento alguno en la resistencia final de la mezcla de concreto, en todas las dosificaciones evaluadas; caso contrario en las mezclas que se evaluaron en este estudio, se observó un incremento en las resistencias finales respecto al diseño planteado. Vale la pena resaltar que el aditivo utilizado en Argentina es a base de Óxido de Calcio y Sulfoaluminato de Calcio, mientras que el utilizado en Colombia es sólo a base de Óxido de Calcio.

Basados en los datos obtenidos mediante esta investigación y comparados con los resultados en Argentina, se podría estar dando el primer paso para crear el concepto de pisos industriales libres de juntas para obtener de este modo un mejor desempeño que los pisos tradicionales, que acarrean muchas más variables como las juntas inducidas, dovelas de trasferencias, sello de juntas, que de no tener un especial cuidado y mantenimiento, llevan a un desgaste prematuro de la estructura. En Colombia el mantenimiento es casi nulo, salvo en estructuras que por sus condiciones así lo demanden.

Se debe tener especial atención en el curado sumergido durante al menos los primeros siete días, ya que algunos procesos químicos propios del aditivo demandan mayor tiempo y disponibilidad de agua para completar su reacción. Si esto no se cumple las propiedades mecánicas de la mezcla se pueden ver disminuidas. Adicionalmente se debe tener en cuenta que este aditivo busca compensar la contracción por secado y por tanto debe controlarse la contracción plástica a través de curado con agua.

Luego de haber realizado pruebas experimentales y comparando los resultados con los expuestos por Collepardi et al. (2008), quienes afirman que es posible realizar losas de grandes dimensiones usando un aditivo compensador de contracción ACC más otros aditivos super-plastificantes, sin realizar curado sumergido. Este tipo de procedimientos tendría un gran incremento en el precio del piso industrial ya que por el momento los aditivos son importados, lo que los hace costosos y comparado con otros métodos usados en la construcción de pisos industriales no sería viable. Sin embargo, se realizó una mezcla de concreto con ACC sin curado (no descritos en este artículo), se 
ensayaron dos vigas a flexión, y los resultados fueron pérdida de resistencia comparados con los que estuvieron sumergidos por un periodo de 7 días. La disminución de MR es del orden de 0.9 a $1 \mathrm{MPa}$ respecto a los curados durante un tiempo de 7 días. Esta prueba demostró además de que luego de ser vaciado no se puede dejar el concreto sin curado, ya que éste es muy importante para que las reacciones químicas en la mezcla de concreto terminen de generarse para obtener los resultados deseados.

\section{Conclusiones}

Se comprobó que la mezcla planteada inicialmente para desarrollar un concreto con un MR de 4.2 MPa tenía un módulo de rotura superior al establecido en el diseño, por lo cual se planteó la reducción en el contenido de cemento de la mezcla de 400 a $380 \mathrm{~kg} / \mathrm{m}^{3}$. Ello representa entre algunas ventajas, tener menor contenido de cemento en la mezcla, motivo por el cual la contracción disminuye e implica menores costos en la elaboración de la mezcla.

De acuerdo con los resultados obtenidos en los ensayos, la dosis óptima de Aditivo Compensador de Contracción ACC es del 9\%, transcurridos 56 días de mediciones, a partir de la elaboración de las mezclas, la variación en el volumen de la masa es cercana a cero, esto es, una variación mínima en el tamaño de la pieza de concreto minimizando los problemas de la contracción por secado.

Al realizar los ensayos de tiempos de fraguado usando ACC, se observó un aumento en dos horas en el fraguado inicial comparado con una mezcla de referencia sin ACC. Esta verificación es muy importante para establecer de forma adecuada los tiempos de desencofre de los especímenes que verifican los cambios de longitud y para tener todas las precauciones del caso durante la producción industrial de CCC.

El ACC probado en los diferentes ensayos genera un aumento en la resistencia de la mezcla de concreto entre un $15 \%$ y un $20 \%$ aproximadamente, comparado con las mezclas realizadas sin ACC. Esta mejora en el desempeño de resistencia corresponde a reacciones químicas presentes en el proceso de hidratación que generan un efecto adherente dentro de la mezcla de concreto. Sin embargo, no debe tomarse este incremento en la resistencia como un elemento para sustituir la cantidad de cemento del diseño original de mezcla por recomendaciones del fabricante, dado que las reacciones del aditivo están relacionadas con la cuantía de cemento presente en la mezcla. Si se modifica la cantidad de cemento en el diseño de mezcla, se deberán hacer nuevamente pruebas que sugieran la dosificación óptima del ACC tipo G con el nuevo diseño.

Los cementos estudiados son conocidos por contener adiciones de residuos de otros procesos industriales. A pesar de las diferencias mineralógicas entre los cementos probados, de los ensayos llevados a cabo se concluye que este aditivo es completamente compatible con los cementos evaluados, lo cual permite recomendarlo como una nueva herramienta para los constructores de pisos industriales que utilicen estos cementos.

Se diseñó exitosamente una mezcla de concreto de contracción compensada para un piso industrial con un MR de 4.2 MPa, comprobado mediante los ensayos de laboratorio realizados, que cumple con todos los estándares de diseño del ACI 360 (2010) y ACI 223 (2010).

Finalmente, al realizar la prueba piloto se observó que la mezcla de concreto a nivel industrial debe monitorearse en cuanto a trabajabilidad y tiempos de fraguado para garantizar continuidad durante el vaciado de concreto.

\section{Recomendaciones}

Se debe consultar la Guía ACI 223 (2010) para las consideraciones de curado sumergido durante al menos 7 días y para todas las consideraciones constructivas a tenerse en cuenta para la aplicación de este tipo de tecnología de losas y concretos con contracción compensada. Se propone un estudio en cuanto a durabilidad del concreto evaluando los componentes activos del ACC y los efectos que puedan llegar a causar a largo plazo en el concreto.

\section{Referencias}

ACI 223 (2010). Guide for the use of shrinkage-compensating concrete. ACI Committee 223R-10, Farmington Hills, MI, USA.

ACI 301 (2010). Specifications for Structural Concrete. ACI Committee 301-10, Farmington Hills, MI, USA.

ACI 302 (2004). Guide for concrete floor and slab construction. ACI Committee 302.1R-04, Farmington Hills, MI, USA. 
ACI 360 (2010). Guide to Design of Slabs-on-Ground. ACI Committee 360R-10, Farmington Hills, MI, USA.

ASTM C150 (2012). Standard Specification for Portland Cement. West Conshohocken, PA, USA.

ASTM C157 (2008). Standard test method for length change of hardened hydraulic-cement mortar and concrete. West Conshohocken, PA, USA.

ASTM C878 (2009). Standard test method for restrained expansion of shrinkage-compensating concrete. West Conshohocken, PA, USA.

Collepardi, M., Troli, R., Bressan, M., Liberatore, F. and Sforza, G. (2008). Crack-free concrete for outside industrial floors in the absence of wet curing and contraction joints. Cement and Concrete Composites 30, Issue 10, 887-891.

Luco, L. F., Pombo, R. and Torrent, R. (2003). Shrinkagecompensating concrete in Argentina. Concrete International 25(5), 91-101.
Mendoza, C. J., Untiveros, A., Máximo, C. y Dávila, P. (2011). Influencia de las fibras de polipropileno en las propiedades del concreto en estados plástico y endurecido. Concreto y cemento: Investigación y Desarrollo 2(2), 35-47.

Monteiro, P.J.M. and Mehta, P.K. (1986). The transition zone between aggregate and type $\mathrm{K}$ expansive cement. Cement and Concrete Research 16(1), 111-114.

Nagataki, S., and Gomi, H. (1998). Expansive admixtures (mainly ettringite). Cement and Concrete Composites 20(2-3), 163-170.

NTC 2871 (2004). Método de ensayo para determinar la resistencia del concreto a la flexión - utilizando una viga simple con carga en los tercios medios. Norma Técnica Colombiana. 